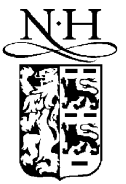

ELSEVIER

\title{
The onset of optical breakdown in KrF-laser-irradiated silica glass surfaces
}

\author{
Y. Kawaguchi ${ }^{\text {a, }}$, A. Narazaki ${ }^{\text {a }}$, T. Sato ${ }^{\text {a }}$, H. Niino ${ }^{\text {a }}$ A. Yabe ${ }^{\text {a }}$, \\ S.C. Langford ${ }^{\mathrm{b}}$, J.T. Dickinson ${ }^{\mathrm{b}}$ \\ ${ }^{a}$ Photoreaction Control Research Center, National Institute of Advanced Industrial Science and Technology, \\ Tsukuba Central 5, 1-1-1, Higashi, Tsukuba, Ibaraki 305-8565, Japan \\ ${ }^{\mathrm{b}}$ Department of Physics, Washington State University, Pullman, WA 99164-2814, USA
}

\begin{abstract}
We have investigated the onset of surface optical breakdown on a polished, synthetic silica glass under KrF excimer laser irradiation. With increasing laser fluence, a transient, localized luminescence is often observed. If the fluence is increased further, the luminescence reappears and grows gradually more intense with continued exposure. Subsequent examination of damaged surfaces show small craters, about $1 \mu \mathrm{m}$ in diameter, in the damaged region. Surfaces damaged by single laser pulses at still higher fluences show similar morphologies, suggesting that the same defects or flaws are responsible for damage under multiple pulse and single pulse conditions.
\end{abstract}

(C) 2002 Elsevier Science B.V. All rights reserved.

Keywords: Optical breakdown; KrF excimer laser; Silica glass; Plume

\section{Introduction}

UV lasers have been widely applied to micro-fabrication processes, for example, in photolithography for CPU manufacture and in hole-drilling for inkjet printer cartridges. Wide bandgap dielectrics, such as fused silica, $\mathrm{CaF}_{2}$ and $\mathrm{MgF}_{2}$, have become important optical materials for manipulating UV laser beams. However, even wide bandgap insulators interact with UV laser light, leading to optical breakdown at fluences above the breakdown threshold or after prolonged exposure at somewhat lower fluences [1]. Improving the UV-resistance of optical materials can significantly enhance the lifetime of micro-fabrication

\footnotetext{
* Corresponding author. Tel.: +81-298-61-4564; fax: +81-298-61-4560.

E-mail address: y-kawaguchi@aist.go.jp (Y. Kawaguchi).
}

systems. Thus it is important to clarify the mechanisms of optical breakdown in these materials. Fused silica is widely used for optical components, and its interaction with UV laser photons has attracted much interest [2$11]$.

In this paper, we investigate the onset of optical breakdown on polished, synthetic silica glass surfaces under single and cumulative $\mathrm{KrF}$ excimer laser irradiation using luminescence spectroscopy, luminescence imaging, and scanning electron microscopy (SEM).

\section{Experimental}

A synthetic fused silica obtained from Tosoh SGM Co., ESL-1000 (OH $\approx 1200$ wt. ppm), with a thickness of $2 \mathrm{~mm}$ was used as the sample. The polishing 
sequence consisted of a rough polish with alumina with a diameter of $23 \mu \mathrm{m}$, a second polish with $\mathrm{CeO}$ with a diameter of $0.5 \mu \mathrm{m}$, and a final polish with colloidal $\mathrm{SiO}_{2}$ with a diameter of $0.08 \mu \mathrm{m}$. The output of a $\mathrm{KrF}$ excimer laser (Lambda Physik COMPEX$102, \lambda=248 \mathrm{~nm}$, pulse width $\approx 20 \mathrm{~ns}$ ) was directed through a variable attenuator (Showa Optronics V248II) and focused onto the sample with a fused silica lens at an angle of about $20^{\circ}$ with respect to the sample normal. Time-resolved luminescence spectra were acquired with a gated optical multichannel analyzer (OMA) in conjunction with a grating spectrometer (JASCO CT-25C, 150 lines $/ \mathrm{mm}$ ), and luminescence images were obtained using a gated ICCD camera. The details of the experimental have been described previously [11-13]. Up to 10 luminescence images were acquired for each laser pulse at a fixed fluence; then the fluence was raised and the same procedure repeated until optical breakdown was observed. The laser-damaged surfaces were observed with an SEM after gold coating.

\section{Results and discussion}

Fig. 1 shows the visible luminescence spectra of silica glass during exposure to single pulses of $\mathrm{KrF}$ radiation at four different fluences. The OMA was triggered $100 \mathrm{~ns}$ after rise of the laser pulse; the spectrum acquisition time was $100 \mu$ s. Prior to the onset of optical breakdown, a second order feature at $496 \mathrm{~nm}$ is observed corresponding to the scattered $248 \mathrm{~nm}$ line. A red emission band centered at $650 \mathrm{~nm}$ is observed that increases linearly with laser fluence. This red band is due to photoluminescence induced at non-bridging oxygen hole centers (NBOHCs; $\left.\equiv \mathrm{Si}-\mathrm{O}^{\bullet}\right)[2,5]$. This red band is a consequence of bond scission in the $\mathrm{Si}-\mathrm{O}$ network to form $\mathrm{NBOHC}$ and $\mathrm{E}^{\prime}$ centers; in our case, these defects are already present in the glass prior to irradiation. It is well known that prolonged exposure to intense $248 \mathrm{~nm}$ irradiation will increase the density of these two defects $[3,4]$.

At a fluence of $3.3 \mathrm{~J} \mathrm{~cm}^{-2}$, an especially bright luminescence is observed, showing the sharp spectral lines characteristic of a plasma associated with surface optical breakdown. All these lines can be assigned to second and third order Si I lines with fundamentals at
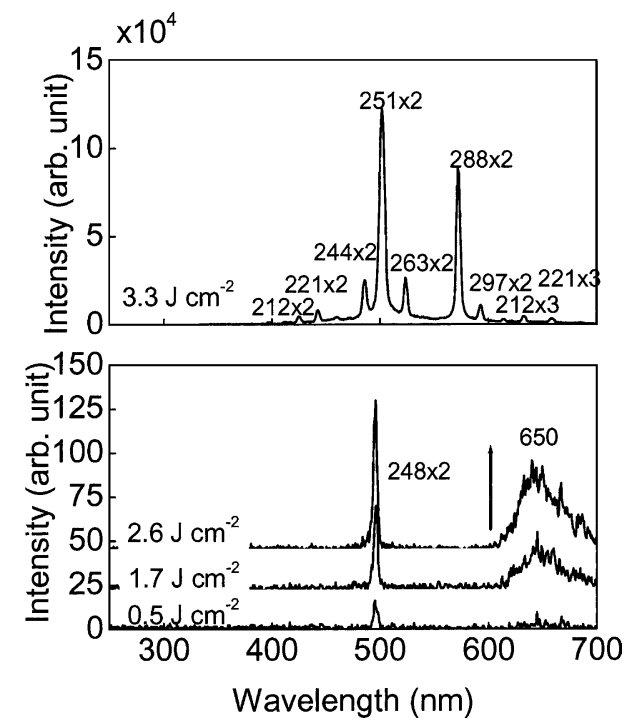

Fig. 1. Fluence dependence of luminescence spectra in silica glass acquired for $100 \mu \mathrm{s}$ in the visible region under irradiation of $\mathrm{KrF}$ laser beam.

$212,221,244,251,263,288$, and $297 \mathrm{~nm}$, due to excited neutral Si atoms [14]. These atomic lines are produced when ground state $\mathrm{Si}$ atoms in the plasma are excited by collisions with energetic electrons [15]. Interestingly, all evidence of line spectra is missing when breakdown is induced inside dielectric samples [16].

Fig. 2(a) shows luminescence images acquired during successive laser pulses on a silica glass surface in a series of exposures. The ICCD camera was triggered $100 \mathrm{~ns}$ after the rise of the $\mathrm{KrF}$ laser pulse, and the acquisition time was set to $1 \mu \mathrm{s}$. Localized luminescence appears on the first pulse after increasing the fluence to $3.2 \mathrm{~J} \mathrm{~cm}^{-2}$. Weaker luminescence is observed at the same location during the second and third pulses. By the fourth pulse, the localized luminescence disappears, leaving only the bulk red luminescence. The appearance and disappearance of localized luminescence was also observed in $\mathrm{NaCl}$ $[12,13]$. We attribute this localized luminescence to micro-plasmas formed when near-surface defective regions are strongly excited by the laser. At this fluence, micro-plume formation apparently removes defective material and possibly anneals nearby glass [11], gradually quenching further luminescence.

When the fluence is further increased to $3.6 \mathrm{~J} \mathrm{~cm}^{-2}$, localized luminescence reappears at the previously 

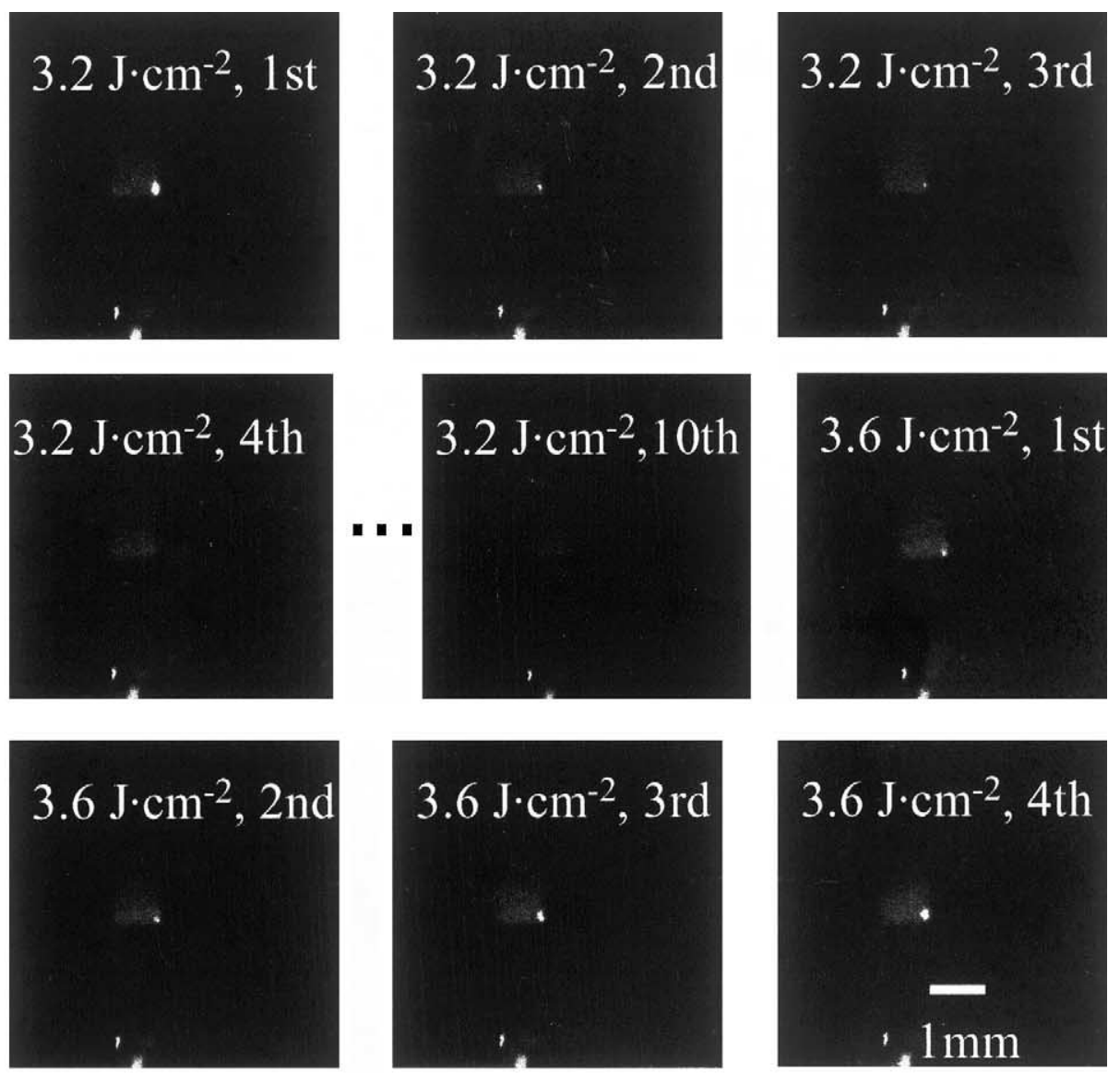

(a)

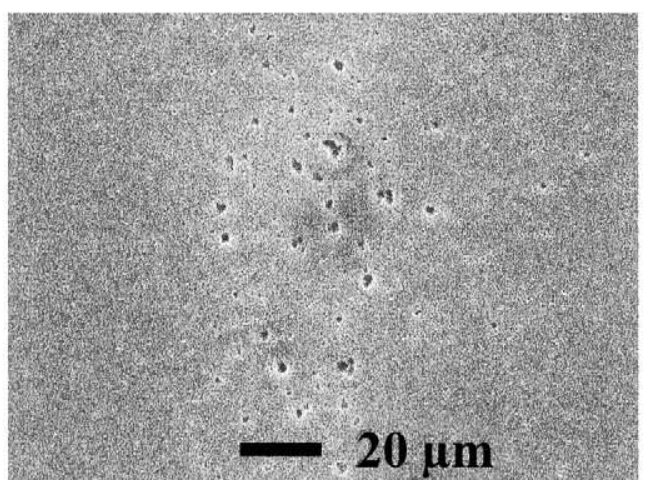

Fig. 2. (a) Luminescence images under irradiation of each laser pulse on the surface of silica glass, (b) SEM image of the laser-damaged region.

damaged spot. At this fluence, the luminescence does not disappear but continues to brighten with each successive pulse. An SEM image of the exposed region is shown in Fig. 2(b). Many small craters, typically several microns in diameter, are observed in the damaged region. These craters represent regions where material has been removed by vaporization due to localized laser absorption and heating [17]. 

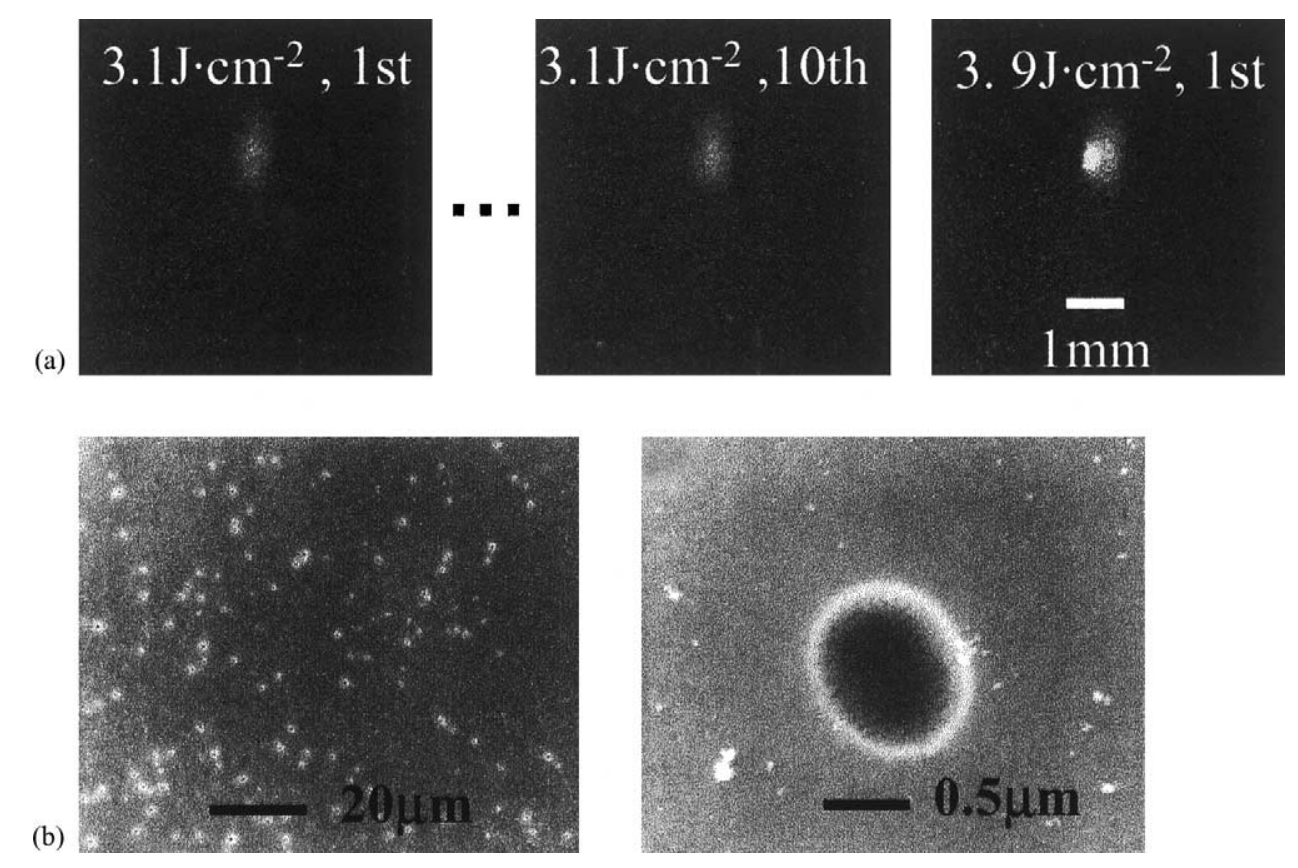

Fig. 3. (a) Another example of luminescence images in silica glass, (b) SEM images of the laser-damaged region with two magnifications.

Vaporized material is required to explain the luminescence spectra. The three laser pulses after the onset of optical breakdown have clearly aggravated the damage created by the initial damaging pulse, enlarging many of the craters and melting nearby material.

Fig. 3(a) shows another set of luminescence images acquired just before and after raising the laser fluence from 3.1 to $3.9 \mathrm{~J} \mathrm{~cm}^{-2}$. The first pulse after increasing the fluence produced bright, localized plume luminescence. SEM images of the laserdamaged region are shown in Fig. 3(b). The pattern of craters is similar to that in Fig. 2(b), but each individual crater is smaller, typically about $1 \mu \mathrm{m}$ in diameter. Additional laser pulses at $3.9 \mathrm{~J} \mathrm{~cm}^{-2}$ presumably would have produced larger craters similar to those in Fig. 2(b).

Fig. 4(a) shows a luminescence image acquired during the first laser pulse incident on an unexposed (a)

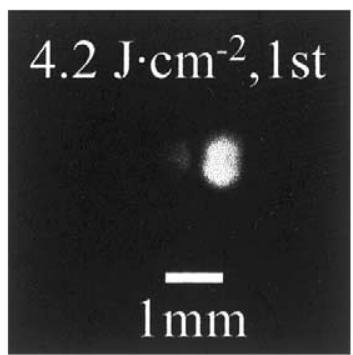

(b)

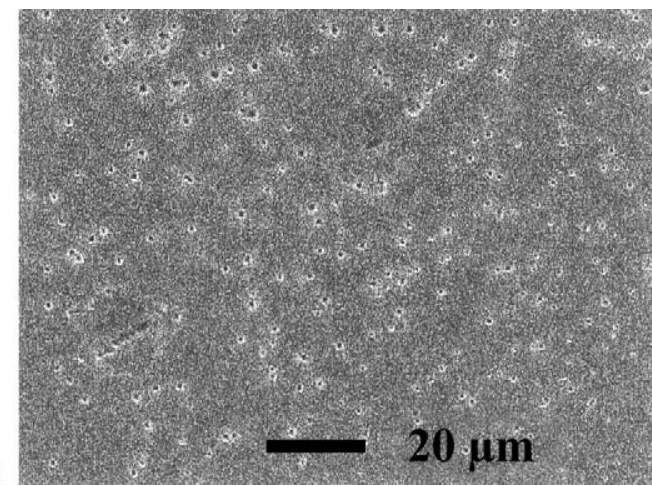

Fig. 4. (a) Luminescence image of silica glass by single-shot irradiation of $\mathrm{KrF}$ laser pulse at $4.2 \mathrm{~J} \mathrm{~cm}^{-2}$, (b) SEM image of the laser-damaged region. 
region of the sample at a fluence of $4.2 \mathrm{~J} \mathrm{~cm}^{-2}$, above the threshold for optical breakdown on these particular surfaces. An SEM image of the region where bright luminescence was observed is shown in Fig. 4(b). The topography of Fig. 4(b) is similar to that in Fig. 2(b), with multiple craters somewhat larger than those produced by single pulses at $3.9 \mathrm{~J} \mathrm{~cm}^{-2}$. Thus a single pulse at $4.2 \mathrm{~J} \mathrm{~cm}^{-2}$ can produce the larger craters that require several damaging pulses at a lower fluence $\left(3.6 \mathrm{~J} \mathrm{~cm}^{-2}\right)$.

To probe the possible role of $\mathrm{OH}^{-}$in the damage process, three synthetic silica glasses were tested, each different $\mathrm{OH}$ contents $(\mathrm{OH}<1 \mathrm{ppm}, \mathrm{OH}=100 \mathrm{ppm}$, and $\mathrm{OH}=1200 \mathrm{ppm})$. All three materials experienced surface breakdown at fluences of $3.3-3.6 \mathrm{~J} \mathrm{~cm}^{-2}$. Further, the morphologies of the resulting damaged surfaces were similar [11]. Although $\mathrm{OH}$ content is known to affect the accumulation of point defects under prolonged irradiation [5,8], $\mathrm{OH}$ has no significant effect on the optical damage morphology observed in this work. Similarly, Sempolinski et al. [6] reported that the changes in absorption under cumulative $\mathrm{KrF}$ irradiation are independent of the $\mathrm{OH}$ content.

The pattern of pit damage observed in this work is similar to patterns of single-shot UV laser damage observed by Yoshiyama et al. [10] on polished fused silica; much of this damage could be eliminated by etching the polished surface to remove the 100$200 \mathrm{~nm}$ surface layer altered by polishing. In optical applications, a chemical etch is not acceptable; thus the effect of polishing on optical damage must be understood. Although the nature of surface layer produced by polishing is not well understood, localized regions of altered material clearly play an important role in the onset of optical breakdown.

The observation of transient plume luminescence under repeated exposure at fluences below the nominal breakdown threshold reflects an incubation process, where damage grows more severe with continued irradiation. On any given sample, we observe an apparent fluence threshold such that micro-breakdown at fluences below a threshold reduces this damage, while micro-breakdown at higher fluences produces further damage that continues to grow with continued irradiation. This threshold fluence corresponds to where laser exposure generates more defects than those removed.

\section{Conclusion}

When polished silica glass is exposed to pulsed $\mathrm{KrF}$ radiation somewhat below the threshold for surface optical damage, transient localized luminescence due to micro-plume formation is observed. Surface optical breakdown itself is accompanied by localized luminescence due to excited neutral $\mathrm{Si}$ atoms, and subsequent SEM images show many small craters about $1 \mu \mathrm{m}$ in diameter in the damaged region. These craters reflect the localized heating and vaporization that give rise to the Si luminescence. The morphology of surfaces damaged by cumulative exposure at fluences below the damage threshold and by single pulses at fluences above the threshold are similar, suggesting that damage under cumulative exposure involves the damage centers responsible for single pulse damage. Further characterization of the interaction of damaged regions with laser radiation may lead to novel strategies for producing more durable, or possibly more fault tolerant, optical components.

\section{Acknowledgements}

This work was supported by the US Department of Energy under Contract DE-FG03-98ER 14864.

\section{References}

[1] R.W. Webb, L.C. Jensen, S.C. Langford, J.T. Dickinson, J. Appl. Phys. 74 (1987) 2323.

[2] R. Tohmon, H. Mizuno, Y. Ohki, K. Sasagane, K. Nakagawa, Y. Hama, Phys. Rev. B 39 (1989) 1337.

[3] K. Arai, H. Imai, H. Hosono, Y. Abe, H. Imagawa, Appl. Phys. Lett. 53 (1988) 1891.

[4] R.A.B. Devine, J. Arndt, Phys. Rev. B 42 (1990) 2617.

[5] D.L. Griscom, J. Ceram. Soc. Jpn. 99 (1991) 923.

[6] D.R. Sempolinski, T.P. Seward, C. Smith, N. Borrelli, C. Rosplock, J. Non-cryst. Solids 203 (1996) 69.

[7] J. Ihlemann, Appl. Surf. Sci. 54 (1992) 193.

[8] N. Kamisugi, N. Kuzuu, Y. Ihara, T. Watanabe, Jpn. J. Appl. Phys. 36 (1997) 6785.

[9] N. Kuzuu, K. Yoshida, H. Yoshida, T. Kamimura, N. Kamisugi, Appl. Opt. 38 (1999) 2510.

[10] J. Yoshiyama, F.Y. Génin, A. Salleo, I. Thomas, M.R. Kozlowski, L.M. Sheehan, I.D. Hutcheon, D.W. Camp, Proc. SPIE 3244 (1998) 331.

[11] Y. Kawaguchi, S.C. Langford, J.T. Dickinson, Proc. SPIE 4274 (2001) 258. 
[12] J.T. Dickinson, Y. Kawaguchi, M.L. Dawes, S.C. Langford, Proc. SPIE 3935 (2000) 38.

[13] Y. Kawaguchi, M.L. Dawes, S.C. Langford, J.T. Dickinson, J. Appl. Phys. 89 (2001) 2370.

[14] D.R. Lide, CRC Handbook of Chemistry and Physics, 81st Edition, CRC Press, Washington, DC, 2000.
[15] D.R. Elmer, S.C. Langford, J.T. Dickinson, J. Appl. Phys. 81 (1997) 1495.

[16] C. Bandis, L.P. Cramer, S.C. Langford, J.T. Dickinson, Appl. Surf. Sci., this issue.

[17] J.T. Dickinson, L.C. Jensen, R.L. Webb, M.L. Dawes, S.C. Langford, J. Appl. Phys. 74 (1993) 3758. 